\title{
Comparación de las propiedades del concreto utilizando escoria de alto horno como reemplazo parcial y total del cemento Pórtland ordinario
}

\author{
L. Espinoza ${ }^{1 *}$ e I. Escalante ${ }^{2}$ \\ ${ }^{1}$ Facultad de Ingeniería Química, Universidad Nacional de Ingeniería (UNI) \\ PO Box 5595, Managua, Nicaragua \\ E-mail: lester.espinoza@gmail.com \\ ${ }^{2}$ Centro de Investigación y de Estudios Avanzados, Instituto Politécnico Nacional (IPN) \\ Unidad Saltillo, Coahuila CP 25900, México \\ E-mail: ivan.escalante@cinvestav.edu.mx
}

(recibido/received: 08-Enero-2007; aceptado/accepted: 28-Mayo-2007)

\begin{abstract}
RESUMEN
Se realizó una investigación con dos tipos de concreto siguiendo la norma ACI 211.1: a) concretos sustituidos al 30, 50 y $70 \%$ con escoria de alto horno (EAH) y b) concretos $100 \%$ EAH activada con 4,6 y $8 \%$ de óxido de sodio $\left(\mathrm{Na}_{2} \mathrm{O}\right)$ en forma de silicato de sodio de módulo 2.0. Todos los sistemas se caracterizaron mecánicamente de 1 a 28 días. Se utilizó microscopía electrónica de barrido y espectroscopía por dispersión de energía para analizar la microestructura, grado de avance de la reacción y productos formados. La sustitución parcial del cemento Pórtland (CPO) por EAH no fue benéfica para las propiedades mecánicas, contrario a la sustitución total por EAH activada, la cuál resultó en propiedades mecánicas superiores que las de concretos de puro CPO. La activación de la escoria con $8 \%$ de $\mathrm{Na}_{2} \mathrm{O}$ resultó más efectiva, seguido de 6 y $4 \%$ de $\mathrm{Na}_{2} \mathrm{O}$.

Palabras claves: activación con silicato de sodio; concreto; escoria activada; escoria de alto horno; propiedades mecánicas del concreto

ABSTRACT

An investigation was carried out with two kinds of concretes following the ACI 211.1 norm: a) replaced concretes with 30, 50 and $70 \%$ with Blast Furnace Slag (BFS) and b) activated $100 \%$ BFS concretes with 4, 6 and $8 \%$ of waterglass $\left(\mathrm{SiO}_{2} / \mathrm{Na}_{2} \mathrm{O}=2.0\right)$ added in equivalent of $\mathrm{Na}_{2} \mathrm{O}$. All the systems were mechanically characterised from 1 to 28 days. Scanning electron microscopy and energy dispersive spectroscopy were used to analyse the microstructure, reaction yield and by-products. The partial substitution of the neat Ordinary Portland Cement (OPC) was not good for the mechanical properties, against the total substitution by activated BFS, which resulted in mechanical properties better than the concretes of neat OPC. The slag activation with $8 \% \mathrm{Na}_{2} \mathrm{O}$ was more effective, followed by 6 and $4 \% \mathrm{Na}_{2} \mathrm{O}$.
\end{abstract}

Keywords: activated slag; activation with waterglass; blast furnace slag; concrete; concrete mechanical properties

*Autor para la correspondencia 


\section{INTRODUCCIÓN}

El concreto es el material que ha tenido el mayor uso en la construcción de edificios e infraestructura en la historia de la civilización. Está constituido principalmente por cemento Pórtland, agregados (gruesos y finos) y agua. Sin embargo, la producción de cemento Pórtland es un proceso que demanda un alto consumo de energía (aproximadamente $4,000 \mathrm{~kJ} / \mathrm{kg}$ cemento, $25 \%$ pérdidas) y con alta emisión de contaminantes $\left(0.85-1.0 \mathrm{~kg}\right.$ de $\mathrm{CO}_{2} / \mathrm{kg}$ cemento) por descarbonatación de materia prima y uso de combustible (Escalante, 2002).

Desde el punto de vista mundial, existe el constante crecimiento de la población, que a su vez demanda infraestructura de vivienda $y$ social. Para satisfacer la creciente demanda de cemento, es necesario encontrar otras alternativas, de otra forma, los costos económicos, energéticos y ambientales serán muy altos.

Es dentro de este contexto que aparece la escoria de alto horno (EAH), la cual es un subproducto no metálico obtenido de la fabricación de hierro. $\mathrm{Su}$ composición química es similar a la del cemento Pórtland, lo cual la hace un material de desecho altamente interesante para su uso como reemplazo parcial o total del cemento Pórtland.

La utilización de la EAH como reemplazo total del CPO implica su activación química con un agente alcalino tal como $\mathrm{NaOH}, \mathrm{Na}_{2} \mathrm{SiO}_{3}$, $\mathrm{Ca}(\mathrm{OH})_{2}, \mathrm{Na}_{2} \mathrm{CO}_{3}, \mathrm{Na}_{2} \mathrm{SO}_{4}$ o mezcla de ellos. $\mathrm{La}$ $\mathrm{EAH}$ al igual que el $\mathrm{CPO}$, tiene propiedades hidráulicas, es decir, al entrar en contacto con el agua ocurren reacciones de hidratación que forman nuevas fases con propiedades cementosas. Sin embargo, la velocidad de reacción de la escoria con el agua es muy lenta, debido a la formación de una capa ácida rica en sílice que impide la penetración del agua hacia el interior de la estructura (Moranville, 1998). Durante la activación química de la escoria, la adición de los iones $\mathrm{OH}^{-}$incrementa la velocidad de reacción facilitando el rompimiento de la estructura vítrea de la escoria y precipitando los productos cementosos (Wang y Scrivener, 1995).
La introducción de la EAH como un nuevo material de construcción no es trivial, especialmente cuando hay vidas humanas que dependen de la solidez de una construcción.

\section{METODOLOGÍA}

\section{Materiales}

Se utilizó cemento Pórtland ordinario (CPO) con una gravedad específica de 3.15 (ASTM C 188$89,1995)$ y un área superficial de $3,250 \mathrm{~cm}^{2} / \mathrm{g}$ (ASTM C204, 1995). La escoria de alto horno (EAH) utilizada provino de la planta Altos Hornos de México, S.A. (AHMSA), ubicada en Monclova, México. Esta escoria tiene una gravedad específica de 2.86 (ASTM C188-89, 1995) y un área superficial de $2,900 \mathrm{~cm}^{2} / \mathrm{g}$ (ASTM C204, 1995).

La composición química del CPO y la EAH utilizada se determinó por microscopía electrónica de barrido acoplada con espectroscopía por dispersión de energía y se presenta en la Tabla 1 (promedio de 8 mediciones):

Tabla 1 Composición química del CPO y la EAH.

\begin{tabular}{lcc}
\hline Óxido $(\% \mathrm{p} / \mathrm{p})$ & CPO & EAH \\
\hline $\mathrm{CaO}$ & 61.58 & 35.69 \\
$\mathrm{SiO}_{2}$ & 22.31 & 36.40 \\
$\mathrm{Al}_{2} \mathrm{O}_{3}$ & 4.90 & 11.22 \\
$\mathrm{SO}_{3}$ & 4.41 & 3.91 \\
$\mathrm{Fe}_{2} \mathrm{O}_{3}$ & 3.87 & 0.64 \\
$\mathrm{MgO}$ & 1.60 & 9.40 \\
$\mathrm{~K}_{2} \mathrm{O}$ & 1.34 & 0.87 \\
$\mathrm{TiO}_{2}$ & - & 1.29 \\
$\mathrm{MnO}$ & - & 0.59 \\
\hline
\end{tabular}

Para la preparación de las mezclas de concreto se utilizó grava de $19 \mathrm{~mm}$, arena de caliza comercializada como No. 5 y agua potable de la zona de Ramos Arizpe, Coahuila, México.

Para la activación química de la escoria se utilizó silicato de sodio $\left(\mathrm{Na}_{2} \mathrm{SiO}_{3}\right)$ grado industrial con una relación en peso $\mathrm{SiO}_{2} / \mathrm{Na}_{2} \mathrm{O}$ de 2.0, a lo que se conoce como el módulo del silicato de sodio. 


\section{Caracterización}

Se fabricaron probetas cúbicas de control (100 $\%$ CPO, de $10 \mathrm{~cm}$ por lado) con una resistencia a la compresión de diseño de $25 \mathrm{MPa}$, a 28 días de curado, siguiendo el proporcionamiento de mezclas de concreto establecido por el American Concrete Institute en su norma ACI 211.1 (1993). Así mismo, se fabricaron probetas de concreto de CPO sustituido con EAH a diferentes niveles $(30,50$ y $70 \%)$ y probetas de concreto $100 \%$ EAH como cementante, activada con $\mathrm{Na}_{2} \mathrm{O}$ en forma de silicato de sodio de módulo 2.0.

En la Tabla 2 se presenta el arreglo experimental de la investigación:

Tabla 2 Arreglo de trabajo.

\begin{tabular}{lcccc}
\hline Sistema & $\begin{array}{c}\mathrm{CPO} \\
(\%)\end{array}$ & $\begin{array}{c}\text { EAH } \\
(\%)\end{array}$ & Agua/MC & $\begin{array}{c}\mathrm{Na}_{2} \mathrm{O} \\
(\% \mathrm{p} / \mathrm{p} \\
\text { respecto a } \\
\text { EAH })\end{array}$ \\
\hline CPO & 100 & 0 & & \\
CE-30 & 70 & 30 & 0.61 & - \\
CE-50 & 50 & 50 & & \\
CE-70 & 30 & 70 & & 4.0 \\
\hline E-40 & 0 & 100 & & 6.0 \\
E-60 & 0 & 100 & 0.45 & 8.0 \\
E-80 & 0 & 100 & & \\
\hline
\end{tabular}

Se fijó como variable controlada que todas las mezclas tuvieran un revenimiento en el cono de Abrams (ASTM C143-90 a, 1995) de $20 \pm 2 \mathrm{~cm}$, ya que se obtiene un mejor manejo del concreto muy bien aceptado en obra.

Todos los sistemas se caracterizaron por ensayo mecánico a $1,3,7,14$ y 28 días de curado en cámara isotérmica a $20 \pm 2{ }^{\circ} \mathrm{C}$. Para cada tiempo de curado se extrajeron aleatoriamente tres probetas de la cámara isotérmica. Los ensayos mecánicos se realizaron en una prensa hidráulica marca D.A.V.I.S.A con una capacidad de 180 toneladas.

Después de ensayadas las probetas, se tomaron muestras representativas. Para analizar las muestras por microscopía electrónica de barrido, se secaron a vacío $\left(40{ }^{\circ} \mathrm{C}\right.$ y $\left.635 \mathrm{~mm} \mathrm{Hg}\right)$ por 24 horas para detener la reacción de hidratación del cementante. El horno de vacío utilizado es marca VWR, modelo 1430M.

Luego del secado, las muestras se montaron en resina epóxica de lento fraguado, se sometieron a vacío para evitar burbujas de aire en su interior y se dejaron fraguar por 24 horas. Después, cada espécimen fue desmoldado, desbastado y pulido con pasta de diamante de hasta $0.25 \mu \mathrm{m}$. Las muestras se recubrieron con grafito previo a su análisis. El equipo utilizado fue un microscopio electrónico de barrido marca Phillips XL 30 ESEM.

\section{RESULTADOS Y DISCUSIÓN}

\section{Resistencia mecánica a la compresión}

En la Tabla 3 se presentan los resultados de resistencia mecánica a la compresión de todos los sistemas. Los valores entre paréntesis corresponden a la desviación estándar para un promedio de tres probetas por cada día de curado.

Tabla 3 Resistencia a la compresión de concretos.

\begin{tabular}{lccccc}
\hline \multirow{2}{*}{ Sistema a } & \multicolumn{5}{c}{ Tiempo de curado (Días) } \\
\cline { 2 - 6 } & 1 & 3 & 7 & 14 & 28 \\
\hline \multirow{2}{*}{ CPO } & 16.55 & 18.98 & 22.85 & 24.53 & 24.61 \\
& $(1.46)$ & $(0.65)$ & $(2.28)$ & $(2.53)$ & $(1.99)$ \\
CE-30 & 11.11 & 11.95 & 15.49 & 15.93 & 20.50 \\
& $(0.28)$ & $(0.61)$ & $(1.08)$ & $(0.91)$ & $(2.18)$ \\
CE-50 & 6.81 & 8.96 & 12.35 & 14.67 & 16.69 \\
& $(0.31)$ & $(0.57)$ & $(0.44)$ & $(1.74)$ & $(0.69)$ \\
CE-70 & 3.67 & 6.40 & 8.65 & 10.91 & 14.36 \\
& $(0.21)$ & $(0.60)$ & $(0.67)$ & $(1.29)$ & $(0.79)$ \\
E-40 & 2.97 & 3.30 & 4.92 & 16.89 & 27.12 \\
& $(0.14)$ & $(0.16)$ & $(0.61)$ & $(0.65)$ & $(1.15)$ \\
E-60 & 8.85 & 10.95 & 20.73 & 30.06 & 32.14 \\
& $(0.39)$ & $(0.89)$ & $(1.17)$ & $(1.67)$ & $(1.57)$ \\
E-80 & 14.41 & 16.16 & 21.49 & 27.86 & 34.98 \\
& $(1.19)$ & $(0.80)$ & $(1.18)$ & $(0.87)$ & $(4.87)$ \\
\hline
\end{tabular}

En la Fig. 1 se presentan los valores de resistencia a la compresión de concretos de $\mathrm{CPO}$ sustituidos con EAH a diferentes niveles. Para 
efectos de comparar, se han insertado los valores de resistencia de concretos $100 \%$ CPO (probetas de control).

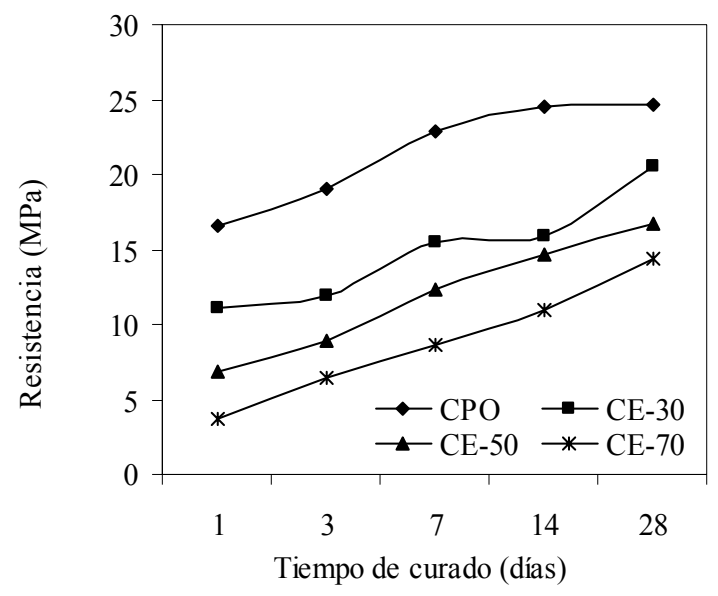

Fig. 1 Resistencia a la compresión de concretos sustituidos con EAH.

Se observa que, la sustitución del CPO con EAH no resultó benéfica para las propiedades mecánicas del concreto. A medida que aumenta el \% de sustitución con EAH, disminuyen las propiedades mecánicas del concreto. En la Fig. 2 se presentan los resultados de resistencia mecánica a la compresión de concretos $100 \%$ EAH activada con álcalis. Para efectos de comparar, se han insertado los valores de resistencia de concretos $100 \%$ CPO (probetas de control).

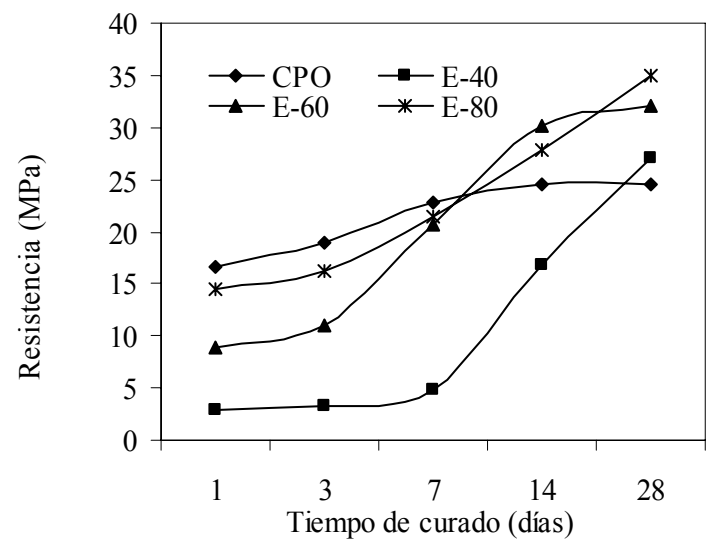

Fig. 2 Resistencia a la compresión de concretos de EAH activada con álcalis.
Se observa que, el desarrollo inicial de resistencia de los concretos $100 \%$ EAH activada es lento en comparación con los concretos 100 $\%$ CPO. Sin embargo, a los 28 días de curado, todos los concretos han alcanzado resistencias mayores a las probetas de control. El desarrollo de resistencia es mayor cuando la EAH se activa con $8 \%$ de $\mathrm{Na}_{2} \mathrm{O}$ y este desarrollo disminuye al disminuir la concentración del agente activante.

\section{Microscopía electrónica de barrido}

Las muestras seleccionadas fueron analizadas a 250X. En la Fig. 3 se presentan las microestructuras para concretos con 0 y $50 \%$ de EAH. A 250X, para una sustitución con $50 \%$ EAH, se puede observar la presencia de EAH parcialmente reaccionada, en contraste para concretos $100 \%$ CPO donde no se aprecian muchos granos de CPO sin reaccionar. Esto indica que la EAH no ha reaccionado lo suficiente para incrementar las propiedades mecánicas y que el CPO ha progresado significativamente en sus reacciones logrando las resistencias a la compresión para las cuales se diseñó el concreto.

La falta de participación de la escoria en las reacciones de hidratación podría ser la causante de la reducción en la resistencia mecánica. En la medida en que la escoria se comporte más cercana a un material inerte químicamente, la comparación entre estos dos concretos sería equivalente a pretender comparar dos concretos de puro CPO, pero uno con 329 y otro con 164.5 $\mathrm{kg} \mathrm{CPO} / \mathrm{m}^{3}$ concreto. Sin embargo, este no es el caso, pues un concreto con $164.5 \mathrm{~kg} \mathrm{CPO} / \mathrm{m}^{3}$ concreto tendría una resistencia del orden de 10 MPa a 28 días (ACI 211.1, 1993) y no de 16.7 $\mathrm{MPa}$ a como se registró. Esto indica que la escoria está participando en las reacciones pero de forma muy limitada.

La Fig. 4 presenta las microestructuras para comparar el efecto del $\%$ de $\mathrm{Na}_{2} \mathrm{O}$ en los concretos de EAH activada. Del lado superior se muestra el concreto activado con $4 \% \mathrm{de}^{\mathrm{Na}_{2}} \mathrm{O}$ y del lado inferior el activado con $8 \%$ de $\mathrm{Na}_{2} \mathrm{O}$. A 250X para $4 \%$ de $\mathrm{Na}_{2} \mathrm{O}$ se observa una mayor cantidad de granos de EAH sin reaccionar con respecto a $8 \%$ de $\mathrm{Na}_{2} \mathrm{O}$. Se puede observar que 
la matriz de productos de reacción del concreto con $4 \%$ de $\mathrm{Na}_{2} \mathrm{O}$ tiene mayor porosidad que la del concreto con $8 \%$ de $\mathrm{Na}_{2} \mathrm{O}$ (mayor cantidad de espacios de color negro).

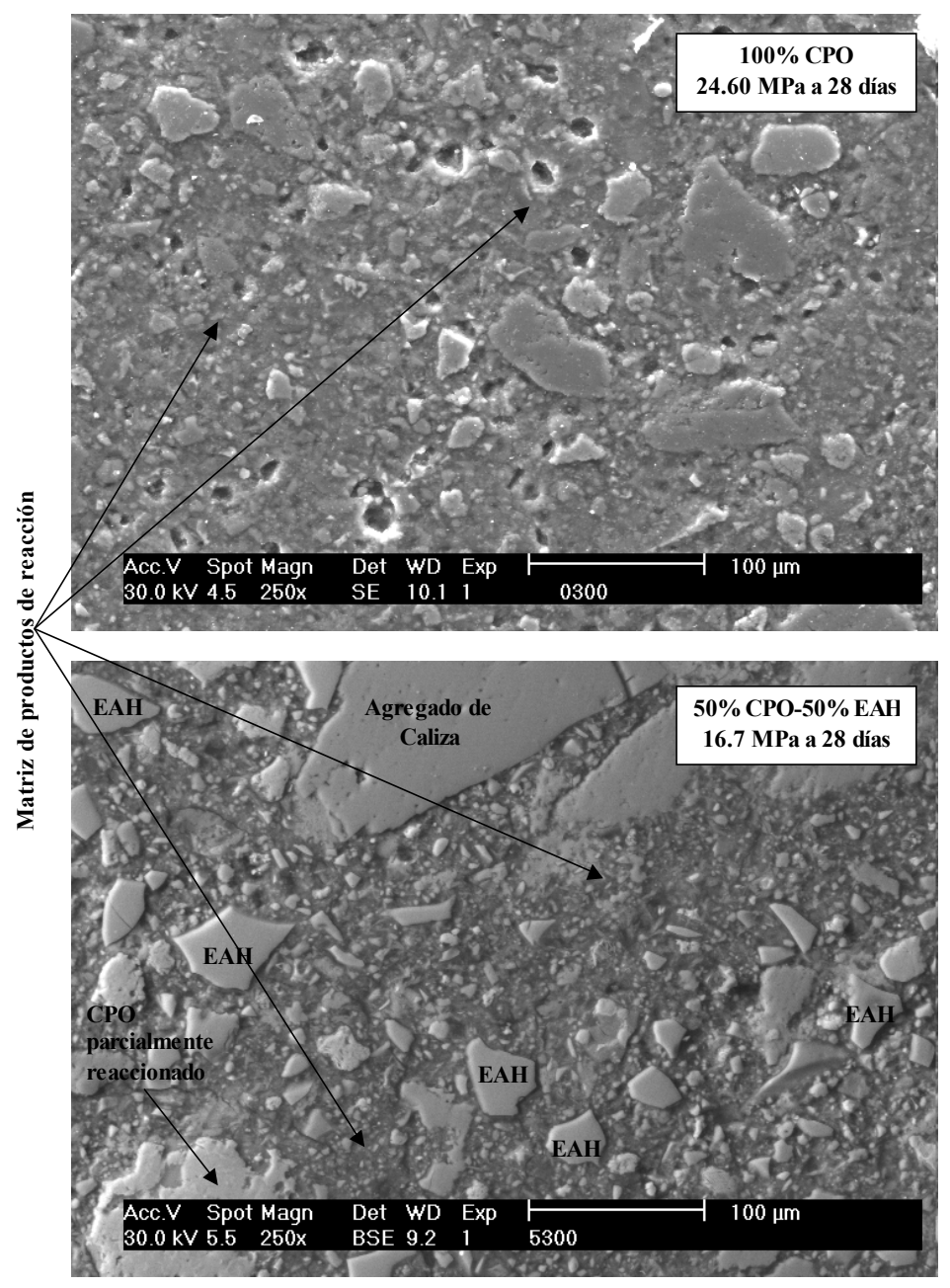

Fig. 3 Microestructura de concreto $100 \%$ CPO (Arriba) y $50 \%$ CPO-50\% EAH (Abajo).

La mayor resistencia del concreto activado con 8 $\%$ de $\mathrm{Na}_{2} \mathrm{O}$ se puede explicar por dos efectos: 1 ) Al incrementar la concentración de $\mathrm{Na}_{2} \mathrm{O}$ se incrementa la intensidad del ataque químico sobre los granos de $\mathrm{EAH}$, lo que resulta en una mayor cantidad de productos de reacción formados y 2) Se incrementa la cantidad de gel de sílice que polimeriza a partir del silicato de sodio. Ambos fenómenos densifican la matriz, disminuyendo la porosidad e incrementando la resistencia a la compresión.

\section{Análisis puntual}

Se realizaron caracterizaciones por análisis puntual en muestras de concreto con $100 \%$
CPO, $50 \%$ EAH-50 \% CPO y $100 \%$ EAH activada con $8 \%$ de $\mathrm{Na}_{2} \mathrm{O}$ en forma de silicato de sodio de módulo 2.0. Se utilizó un microscopio electrónico de barrido Phillips XL 30 ESEM acoplado con un microanalizador EDAX modelo Falcon. El tiempo de cada análisis fue de 50 segundos.

Los análisis puntuales se realizaron en la matriz de productos de hidratación y se tomaron 30 microanálisis para cada muestra de concreto. Los resultados se muestran en relaciones de composición $\mathrm{Al}_{2} \mathrm{O}_{3} / \mathrm{CaO}$ vs. $\mathrm{SiO}_{2} / \mathrm{CaO}$ como se reportan comúnmente para sistemas de cemento Pórtland y escoria activada (Fraire, 2001). 


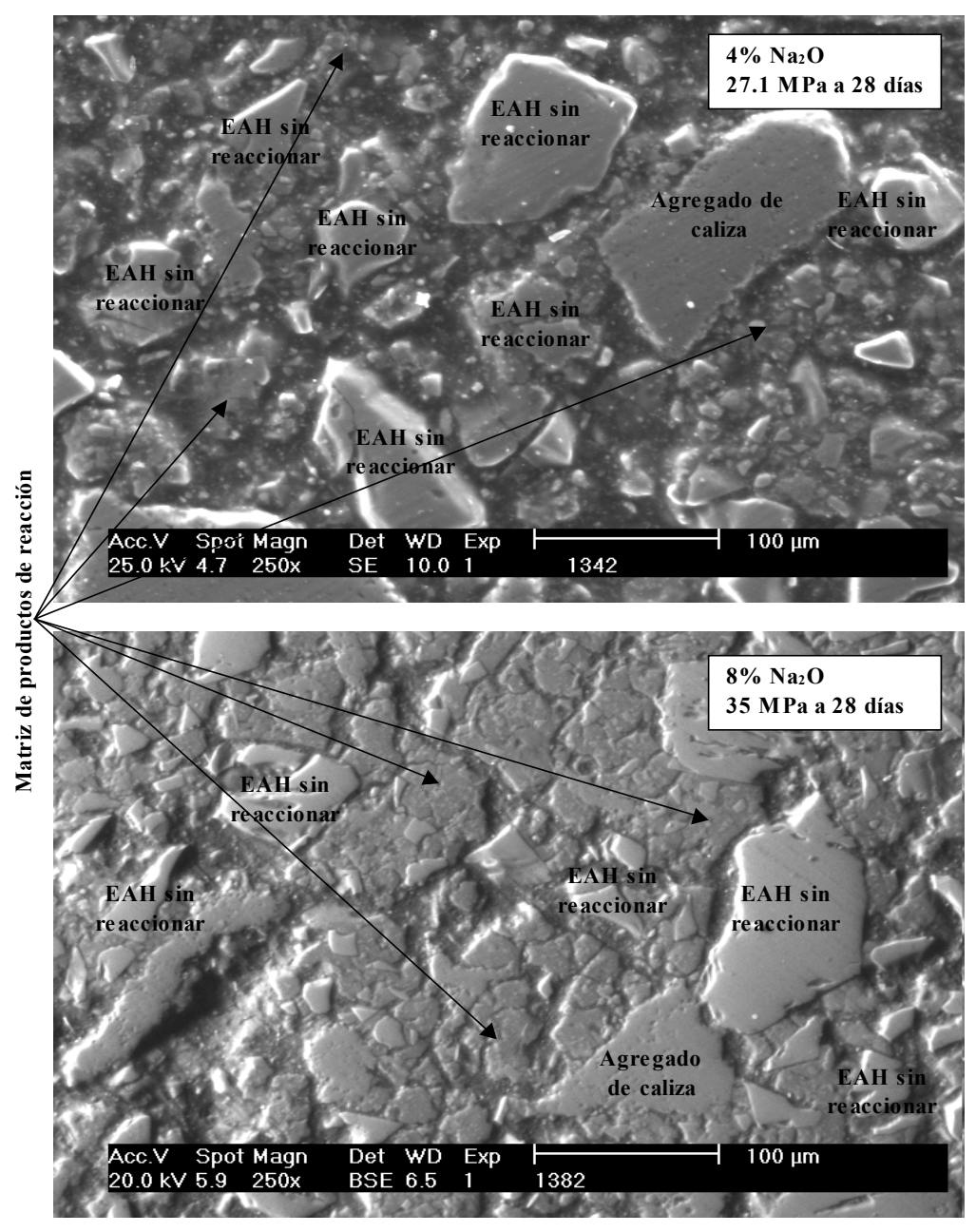

Fig. 4 Microestructura de concreto $100 \%$ EAH activado con $4 \%$ y $8 \%$ de $^{N_{2}} \mathrm{O}_{2}$.

En la Fig. 5 se muestran los resultados para el concreto $100 \% \mathrm{CPO}$; en ella se observa que la composición de los productos de reacción es dispersa. Sin embargo, considerando los productos de la reacción de hidratación del CPO es posible entender mejor la gráfica; para esto se incluyen las composiciones teóricas del $\mathrm{AFt}$ (Trisulfoaluminato de calcio hidratado), AFm (Monosulfoaluminato de calcio hidratado) y $\mathrm{Ca}(\mathrm{OH})_{2}$ (Portlandita). Se puede notar que existe un grupo de puntos cuya composición se agrupa en una relación $\mathrm{SiO}_{2} / \mathrm{CaO}$ entre 0.40 y 0.50 , los cuales corresponderían al C-S-H $\left(\mathrm{C}_{2.22}-\mathrm{S}-\mathrm{H}\right)$.

Así mismo, si se trazan arbitrariamente líneas entre el grupo de datos del C-S-H y las composiciones teóricas antes citadas, puede notarse que algunos puntos caen en tales líneas. Por ejemplo, puede decirse que los análisis que caen a lo largo de la línea C-S-H--Ca(OH) corresponden a una mezcla de ambos compuestos.

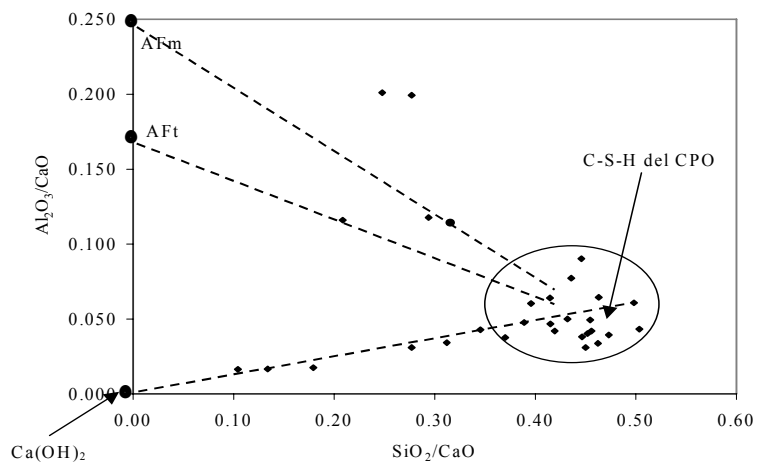

Fig. 5 Análisis puntual del concreto $100 \%$ CPO. 
La Fig. 6 presenta los resultados del microanálisis para el concreto $50 \%$ CPO-50\% EAH. Los resultados muestran dispersión como los productos de $100 \%$ CPO. Sin embargo, puede notarse que la composición química es en general diferente. El C-S-H del cemento sustituido tiene una relación $\mathrm{SiO}_{2} / \mathrm{CaO}$ entre 0.40 y 0.65 , que es mayor que la del CPO puro. Esto indica una menor presencia de $\mathrm{CaO}$ en los productos C-S-H del CPO-EAH; la escoria tiene menos $\mathrm{CaO}$ que el $\mathrm{CPO}$ y para satisfacer esta deficiencia y formar C-S-H reacciona con el $\mathrm{Ca}(\mathrm{OH})_{2}$ formado por el CPO y descalcifica al C-S-H formado por la fracción del CPO. Puede notarse que para este concreto no hay puntos entre el C-S-H y la composición teórica del $\mathrm{Ca}(\mathrm{OH})_{2}$, lo cual indica que este ha sido consumido al no aparecer en los productos de reacción.

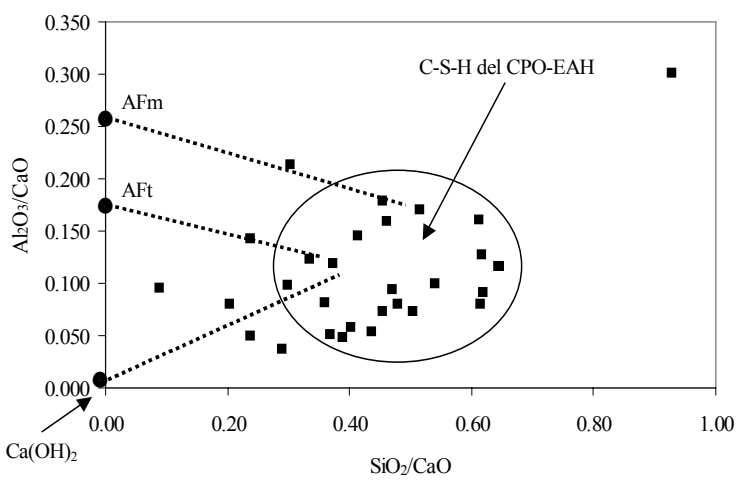

Fig. 6 Análisis puntual para el concreto $50 \%$ CPO-50 \% EAH.

En la Fig. 7 se presentan los resultados obtenidos para el concreto $100 \%$ EAH activada con $8 \%$ de $\mathrm{Na}_{2} \mathrm{O}$. Las diferencias en las composiciones de los productos de reacción entre los diferentes sistemas cementantes son claras. La relación $\mathrm{SiO}_{2} / \mathrm{CaO}$ está entre 1.20 y 1.78 con un promedio de 1.49. Escalante et al. (2002) reportó un promedio de 1.38 (con valores entre 1.22 y 1.53 ) para pastas de escoria activada con $5 \%$ de silicato de sodio de módulo 2.0. La relación $\mathrm{SiO}_{2} / \mathrm{CaO}$ obtenida para concretos de EAH activada es alrededor de 4 veces mayor que la obtenida para CPO y CPO-EAH. Esto se debe a los altos contenidos de $\mathrm{SiO}_{2}$ y bajos contenidos de $\mathrm{CaO}$ de la EAH, relativo al CPO. Estos datos indican que la hidratación de la escoria ha avanzado notablemente. Además, el $\mathrm{SiO}_{2}$ agregado al concreto en forma de silicato de sodio aumenta esta relación.

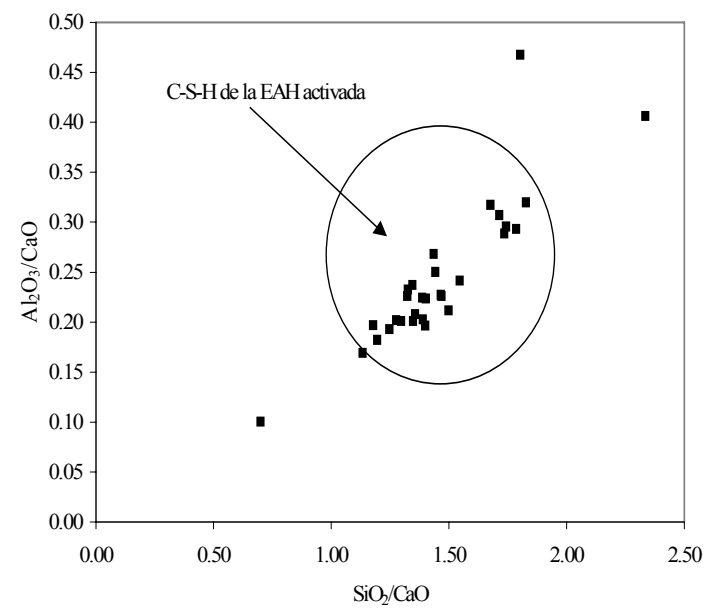

Fig. 7 Análisis puntual par el concreto $100 \%$ EAH activada con álcalis.

\section{CONCLUSIONES}

Ninguno de los concretos de cemento compósito (CPO-EAH) alcanzó resistencia mecánica a la compresión mayor que la de los concretos de puro CPO. La sustitución parcial del CPO por $\mathrm{EAH}$ no fue favorable para las propiedades mecánicas. La resistencia a la compresión disminuyó conforme se aumentó el \% de sustitución con EAH. Esto se debió a una limitada participación de la escoria en las reacciones de formación de $\mathrm{C}-\mathrm{S}-\mathrm{H}$.

Los concretos $100 \%$ EAH activados con álcalis alcanzaron resistencias mayores que los concretos $100 \%$ CPO. Los concretos que alcanzaron las mayores resistencias $y$ a velocidades mayores fueron aquellos activados con $8 \%$ de $\mathrm{Na}_{2} \mathrm{O}$, seguido de los activados con 6 y $4 \%$ de $\mathrm{Na}_{2} \mathrm{O}$. A mayor concentración de $\mathrm{Na}_{2} \mathrm{O}$, el ataque químico sobre la escoria es más intenso dando como resultado una mayor cantidad de productos de hidratación que densifica la matriz aumentando la resistencia mecánica. El principal producto de hidratación encontrado es el silicato de sodio hidratado (C$\mathrm{S}-\mathrm{H}$ ), aunque con diferente composición entre los diferentes sistemas cementantes (CPO, CPOEAH, EAH activada). 


\section{AGRADECIMIENTOS}

Los autores agradecen a la Universidad Nacional de Ingeniería, al Centro de Investigación y Estudios Avanzados, al Consejo Nacional de Ciencia y Tecnología, al proyecto Coahuila No. 403 y a la Secretaría de Medio Ambiente y Recursos Naturales, por el apoyo y financiamiento para la realización de este trabajo.

\section{REFERENCIAS}

ACI 211.1. (1993). Proporcionamiento de Mezclas de Concreto (concreto normal, pesado $y$ masivo), pp. 21-28. México D.F, México.

ASTM C143-90 a. (1995). Test Method for Slump of Hydraulic Cement Concrete. Annual book of ASTM standards, Volume 04.02, concretes and aggregates. Philadelphia, USA.

ASTM C188-89. (1995). Standard Test Method for Density of Hydraulic Cement Concrete. Annual book of ASTM standards, Volume 04.01, cement, lime and gypsum. Philadelphia, USA.

ASTM C204. (1995). Standard Test Method for Fineness of Hydraulic Cement by Air Permeability Apparatus. Annual Book of ASTM Standards, Volume 04.01, cement, lime and gypsum. Philadelphia, USA.

Escalante, J.I. (2002). Materiales Alternativos al Cemento Pórtland. Avance y Perspectiva. Vol. 21, pp. 79-88.

Escalante, J.I., J.M. Nonell, A. Gorokhovsky, P.E. Fraire, H.M. Molinar y G.M. Suárez (2002). Reactividad y Propiedades Mecánicas de la Escoria de Alto Horno Activada por Álcalis. Boletín de la Sociedad Española de Cerámica y Vidrio. Vol. 41, pp. 451-458.

Fraire, P.E. (2001). Propiedades Mecánicas y Reactividad de la Escoria de Alto Horno Activada por diferentes Agentes Alcalinos. Tesis de Maestría en Ciencias en Ingeniería Cerámica. CINVESTAV del IPN. Saltillo, México.
Moranville, M. (1998). Chemistry of Cement and Concrete. $4^{\mathrm{TH}}$ Edition. pp. 637-673. Editorial Elsevier. Londres, Inglaterra.

Wang, S.D. y K.L. Scrivener (1995). Hydration Products of Alkali Activated Slag Cement. Cement and Concrete Research. Vol. 25, No. 03, pp. 561-571.

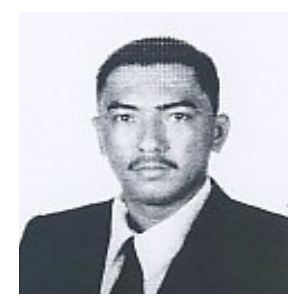

Léster Espinoza se graduó de Ingeniero Químico en la Universidad Nacional de Ingeniería (UNI) en el 2006. Realizó su tesis de graduación en el Centro de Investigación y Estudios Avanzados de México en el área de Ingeniería Cerámica bajo la dirección del Dr. Iván Escalante.

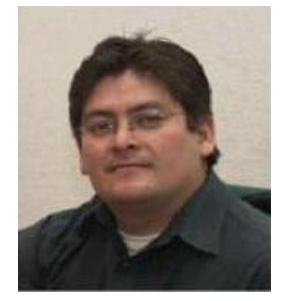

Iván Escalante se graduó de Doctor $(\mathrm{PhD})$ en la Universidad de Sheffield, Reino Unido en 1996. Su línea de investigación es el desarrollo de nuevos materiales cementosos sustitutos parciales o totales del cemento Pórtland. Profesor Investigador titular del Centro de Investigación y Estudios Avanzados e Investigador Nivel II del Sistema Nacional de Investigadores de México. 